LA-UR-01-1617

Approved for public release; distribution is unlimited.

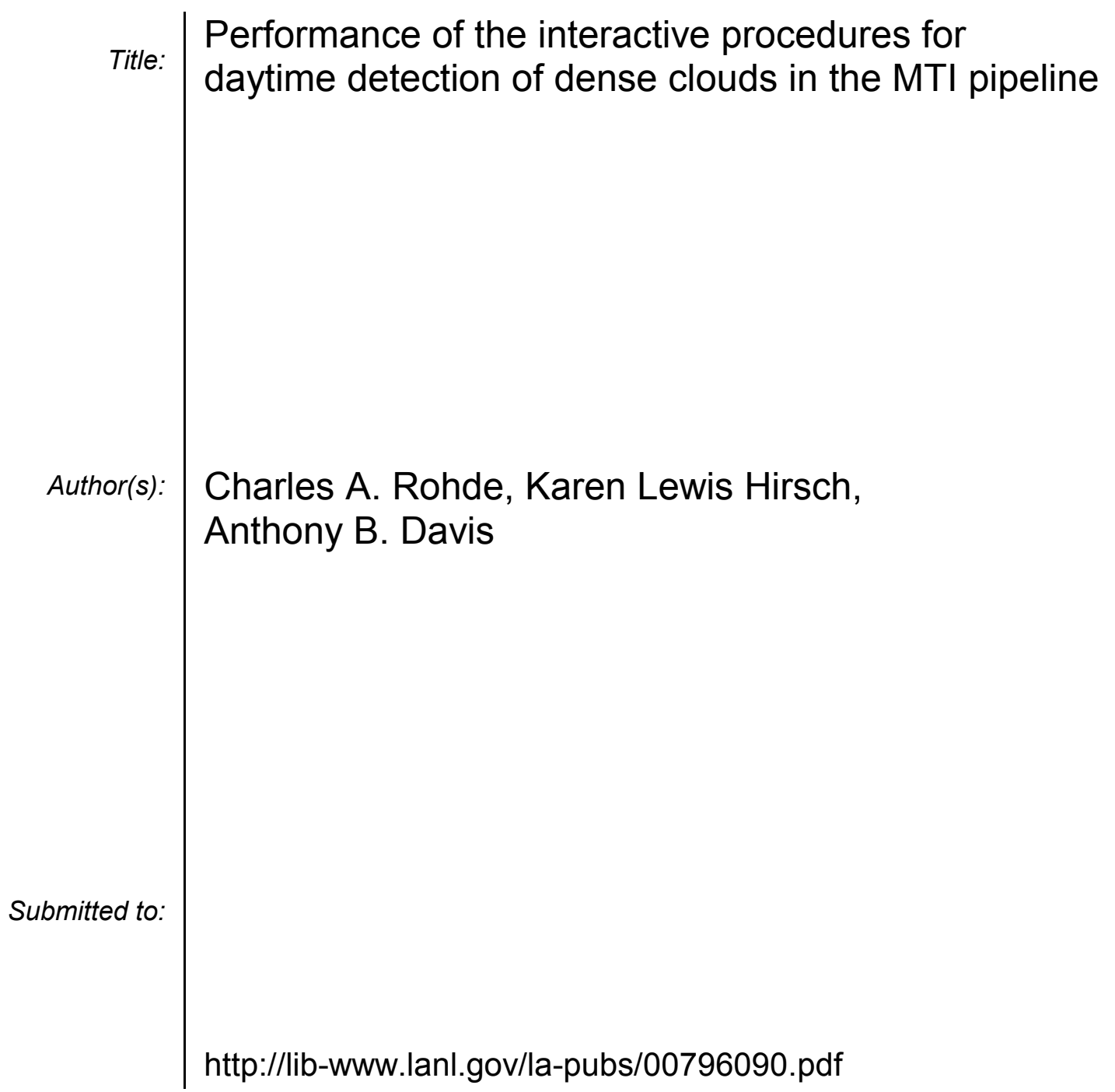

Los Alamos National Laboratory, an affirmative action/equal opportunity employer, is operated by the University of California for the U.S. Department of Energy under contract W-7405-ENG-36. By acceptance of this article, the publisher recognizes that the U.S. Government retains a nonexclusive, royaltyfree license to publish or reproduce the published form of this contribution, or to allow others to do so, for U.S. Government purposes. Los Alamos National Laboratory requests that the publisher identify this article as work performed under the auspices of the U.S. Department of Energy. Los Alamos National Laboratory strongly supports academic freedom and a researcher's right to publish; as an institution, however, the Laboratory does not endorse the viewpoint of a publication or guarantee its technical correctness. 


\title{
Performance of the interactive procedures for daytime detection of dense clouds in the MTI pipeline
}

\author{
Charles A. Rohde, Karen Lewis Hirsch, Anthony B. Davis \\ Los Alamos National Laboratory \\ Space and Remote Sensing Sciences Group (NIS-2) \\ P.O. Box 1663 (Mail Stop C-323) \\ Los Alamos, NM, 87545, USA
}

\begin{abstract}
Pixel-scale cloud detection relies on the simple fact that dense-enough clouds are generally brighter, whiter, and colder than the underlying surface. These plain-language statements are readily translated into threshold operations in the multispectral subspaces, thus providing a reasonable premise for searching data cubes for cloud signatures. To supplement this spectral input (VIS, NIR, and TIR channels), we remark that cloud tops are generally above most of the water vapor in the atmosphere column. An extra threshold in the MTI water vapor product can therefore be applied. This helps considerably in cases where one of the default cloud signatures becomes ambiguous. The resulting cloud mask is however still sensitive to the thresholds in brightness, whiteness, temperature, and column water content, especially since we also want to flag low-level clouds that are not-so-dense. Clouds are also generally spatially large compared to pixel size. This implies that simple spatial morphological filters can be use to remove false positives and for expansion of the cloud mask. A false positive is indeed preferable to a miss in the view of MTI's mission in support of nuclear non-proliferation; non-local cloud radiative effects can otherwise bias retrievals in adjacent cloud-free areas. Therefore we use a data analyst to ensure builtin quality control for MTI cloud masks. When looking for low-level clouds, the analyst interacts with a GUI containing histograms, a customized RGB rendering of the input data, and an RGB diagnostic cloud mask for quick evaluation of all threshold values. We use MTI data to document the performance and analyst-sensitivity to this procedure.
\end{abstract}

Keywords: MTI, Multispectral Imaging, Cloud Detection

\section{INTRODUCTION}

The Multispectral Thermal Imager (MTI) Satellite ${ }^{1}$ is a state-of-the-art multispectral imaging platform. Its development and use are joint projects between Los Alamos National Laboratory, Sandia National Laboratory and Savannah River Technology Center. Cloud detection is an important factor in both MTI's primary non-proliferation mission of accurate surface temperature retrieval and its secondary environmental studies mission. In the former it is important to remove the aberrations clouds produce in order to achieve desired surface temperature accuracy. In the latter mission of environmental study, clouds become important in their own right, primarily as agents of climatological change. In this case a general cloud mask is useful for later classification schemes.

MTI has 15 spectral bands ranging from visible wavelengths $(450 \mathrm{~nm})$ to longwave infrared $(10 \mu \mathrm{m}$, Fig. 1$) .^{2}$ This selection of bands allows for a simple translation of a cloud's general characteristics into spectral band operations. Clouds are (generally): bright at visible wavelengths, evenly reflective at many wavelengths from visible to nearinfrared, higher in the atmosphere than most image features, and hence, colder and drier than most image features. The selection of a threshold on a visible and a longwave infrared band comes directly from the first two cloud characteristics. While the creation of NVDI and CIBR like band ratios can serve as measures of the whiteness and dryness conditions. Another general characteristic of a dense cloud is a large spatial extent. There are several types of spatial filtering that effectively sort large and small features. We choose to implement a morphological opening of variable size in order to remove image residues of small size that pass through the spectral threshold filtering.

We will be discussing how these ideas have been implemented into the creation of MTI cloud masks. Both the algorithm and the analyst GUI are individually described. Finally a comparison of analyst response is given. These

Send correspondence to carohde@lanl.gov, Tel: +1-505-665-5481; Fax: +1-505-667-9208 


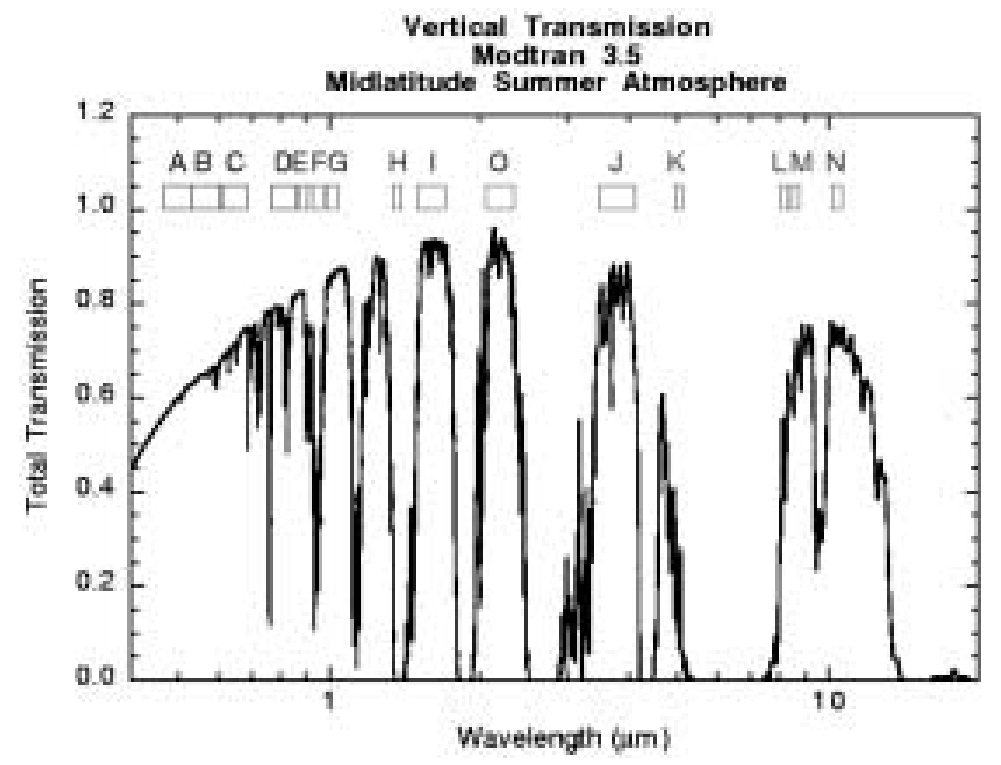

Figure 1. MTI spectral bands plotted upon a atmospheric transmission plot. Reproduced from Clodius et al.. ${ }^{2}$ The MTI cloud mask uses bands $\mathrm{C}$ (brightness threshold), $\mathrm{E}$ (used with $\mathrm{C}$ to form a NDVI like ratio, for the whiteness threshold), F \& G (used with $\mathrm{E}$ to form CIBR water vapor product to form the dryness threshold), and $\mathrm{N}$ for temperature thresholding.

results show there is little variation in the user responce. We have compared elsewhere ${ }^{3}$ the performance of a simpler version of our new interactive (hence highly data-adaptive) scheme to other cloud mask algorithms, namely, a genetic programming approach ${ }^{4}$ and a sophisticated location-sensitive approach developed for MODIS/Terra-Aqua. ${ }^{5}$

\section{ALGORITHM}

\subsection{Spectral thresholding}

The MTI Cloud Mask spectral thresholding algorithm is straightforward and simple. Using a visible band (C: 620-680 $\mathrm{nm})$ a threshold for minimum brightness is selected. Every pixel above this value is marked true in the brightness mask $(B M)$. Also using a single longwave infra-red band (N: 10.2-10.7 $\mu \mathrm{m}$ ) an upper limit on temperature is selected. All pixels less than this temperature are marked true in the temperature mask $(T M)$. To determine the whiteness of a pixel, bands E (860-890 nm) and C are used in an NDVI like ratio. An upper limit on uneven reflectivity can be established using:

$$
\left|\frac{E-C}{E+C}\right|
$$

The use of the absolute values makes this slightly different from the standard NDVI. Evenly reflecting (white) objects will have NDVI values around zero. Using the absolute values allows for specifying only an upper limit, instead of a range. Any values less than this upper limit are marked as true in the whiteness mask $(W M)$. We then construct an image whose values vary monotonically as a function of the column integrated water vapor content using bands, E, F (910-970 nm), and G (990-1040 nm) to form a weighted Continuum Interpolated Band Response (CIBR) ratio ${ }^{6}$;

$$
\frac{F}{w_{1} G+w_{2} E} \text {. }
$$

The $\mathrm{F}$ band is centered on a water vapor absorption feature. The above ratio is therefore largest when the column integrated water vapor is smallest. We therefore pick a dryness threshold and mark any pixel value greater as true in a dryness mask $(D M)$. 
Since not every cloud will be the brightest, whitest, coldest and driest set of pixels in every image, we have incorporated a "best of $\chi$ " scheme in oder to loosen the obligation placed on the analyst to find the perfect threshold values. This manifests itself by selecting true pixels for the final binary cloud mask from the criteria that, the sum of the four spectral masks must be greater than or equal to the parameter $\chi$. The basic spectral thresholding can be summarized as

$$
\begin{gathered}
B M_{i, j}=\left\{\begin{array}{l}
1, C_{i, j} \geq \beta \\
0, C_{i, j}<\beta
\end{array}, W M_{i, j}=\left\{\begin{array}{l}
1, \mid \frac{E_{i, j}-C_{i, j}}{E_{i, j}+C_{i, j}} \leq \omega \\
0,\left|\frac{E_{i, j}-C_{i, j}}{E_{i, j}+C_{i, j}}\right|>\omega
\end{array}, \quad T M_{i, j}=\left\{\begin{array}{l}
1, N_{i, j} \leq \tau \\
0, N_{i, j}>\tau
\end{array}, \quad D M_{i, j}=\left\{\begin{array}{l}
1, \frac{F_{i, j}}{w_{1} E_{i, j}+w_{2} G_{i, j}} \geq \delta \\
0, \frac{F_{i, j}}{w_{1} E_{i, j}+w_{2} G_{i, j}}<\delta
\end{array}\right.\right.\right.\right. \\
C M_{i, j}=\left\{\begin{array}{l}
1, B M_{i, j}+W M_{i, j}+T M_{i, j}+D M_{i, j} \geq \chi \\
0, B M_{i, j}+W M_{i, j}+T M_{i, j}+D M_{i, j}<\chi
\end{array} .\right.
\end{gathered}
$$

$\beta$ being the brightness threshold, $\omega$ the whiteness threshold, $\tau$ the temperature threshold, and $\delta$ the dryness threshold. In addition to selecting the $\chi$ parameter, the analyst may also select specific mask combinations, overriding the $\chi$ parameter, on a scene-by-scene basis. This is discussed further in section 2.7 below.

\subsection{Spatial filtering}

Clouds that are that are dense enough to effect radiometry retrievals are also typically large in spatial extent. Some smaller clouds are dense enough to see, but are still very similar to other scene values. When attempting to create conservative cloud masks, small artifacts (on the order of a few pixels) appear in the binary mask. In order to find all of the clouds in an image it is necessary to accept these false positives.

In Hirsch et al., ${ }^{3}$ GENIE ${ }^{4}$ a computer learning program, was applied to the problem of cloud identification in simulated MTI images. The solution that GENIE found, was similar to the thresholding scheme presented in this paper. However, in addition to spectral filtering, GENIE also chose to use spatial morphological filters. On the basis of these results, spatial filtering was incorporated into the MTI cloud mask algorithm.

Two of the basic filters used were erode and dilate filters. An erosion filter is a neighborhood minimum operator. ${ }^{7}$ An array of values, called the structuring element, is centered on the pixel of interest. The minimum of the logical AND of the structuring element and the image then replaces the current pixel value. When using a square structuring element of ones on a binary cloud mask, this affectively removes any isolated mask artifacts smaller than the size of the filtering structure. The dilate filter is a neighborhood maximum operator. This operator allows for growing of the binary cloud mask boundaries. Thus allowing an analyst to capture clouds boundaries that can often be difficult to capture with minimal false positive response only using thresholds.

When used with the same structuring element, a dilate followed by an erode is a new morphological filter called a closing. This is because it has the effect of closing holes smaller than the structuring element in binary cloud masks without further effecting the cloud mask. Often this is very useful from a retrieval standpoint. If a cloud completely surrounds a small hole, the retrieval of values from the hole is more than likely biased. These holes should therefore also be flagged as cloud.

Used together, these three spatial filters are used to post-process the spectral filtering. The results are cloud masks with fewer false positives, the ability to "grow" a cloud mask to completely cover cloud edges, and the possibility of filling in holes in the mask.

\subsection{A GUI approach to spectral/spatial cloud mask production}

In order to keep results consistent and the work load on the human analyst small, an approach to spectral and spatial filtering is taken that is pragmatic and simple. A graphical user interface (GUI) was built using IDL, to assist an analyst with the spectral threshold selection. This GUI has several features that are specific to helping the analyst create accurate and consistent cloud masks.

Figure 2 shows an example session of the interactive cloud mask creation GUI. In the input window, the analyst is presented with an RGB image; the red channel is set to band $\mathrm{N}$, the green channel to band $\mathrm{E}$, and the blue channel is the $\mathrm{C}$ band. This image's intensity is then normalized with the CIBR water vapor ratio. This scheme creates bright cyan clouds against a less intense, redder background. This RGB image may be viewed any time during the cloud mask creation process. 


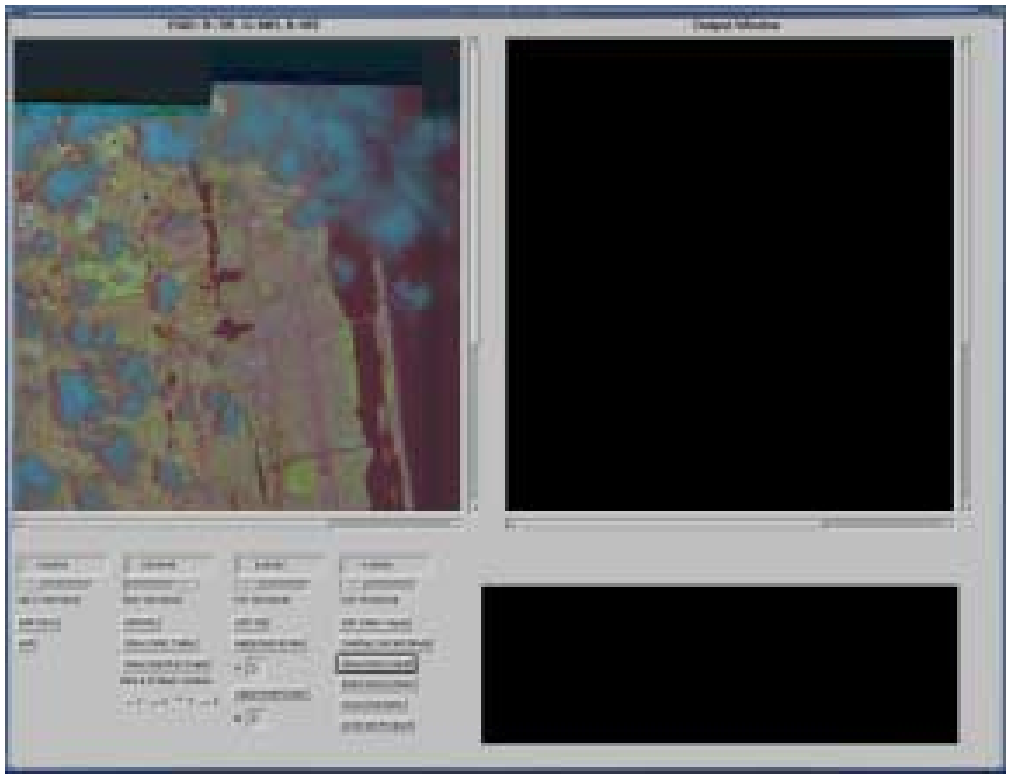

Figure 2. GUI interface, initial RGB input image. $\mathrm{R}=$ band $\mathrm{N}, \mathrm{G}=$ band $\mathrm{E}, \mathrm{B}=$ band $\mathrm{E}$ with intensity normalized by CIBR as a measure of water-vapor content.

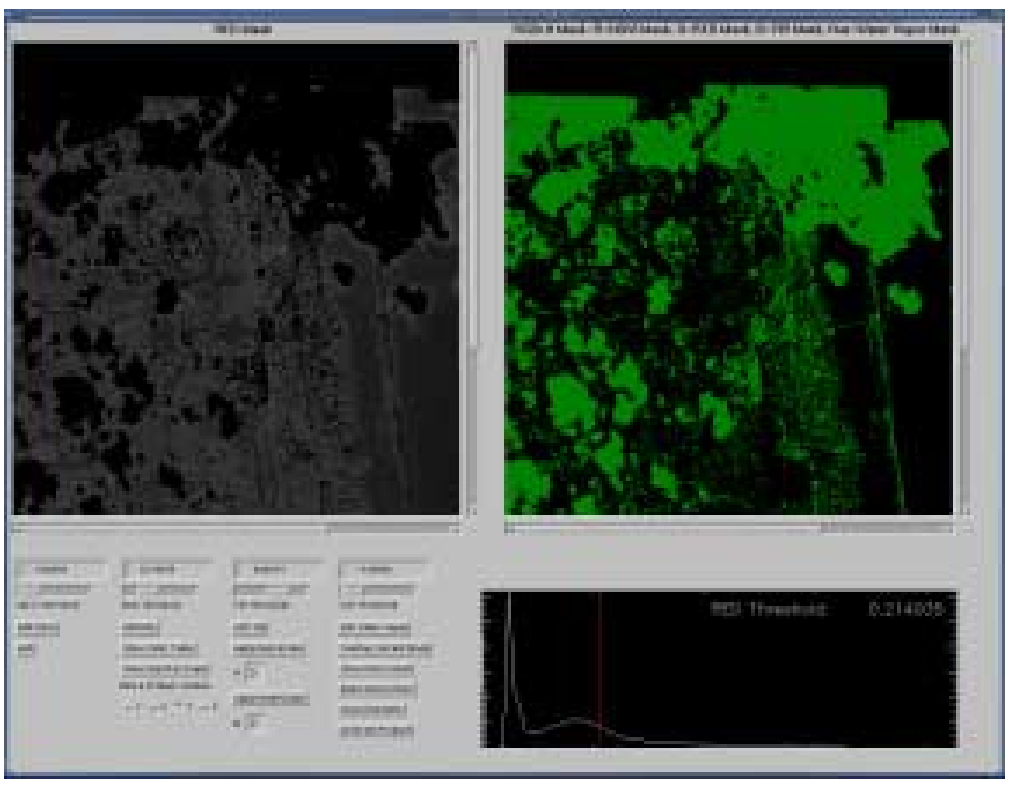

Figure 3. Mask creation for an individual threshold. 


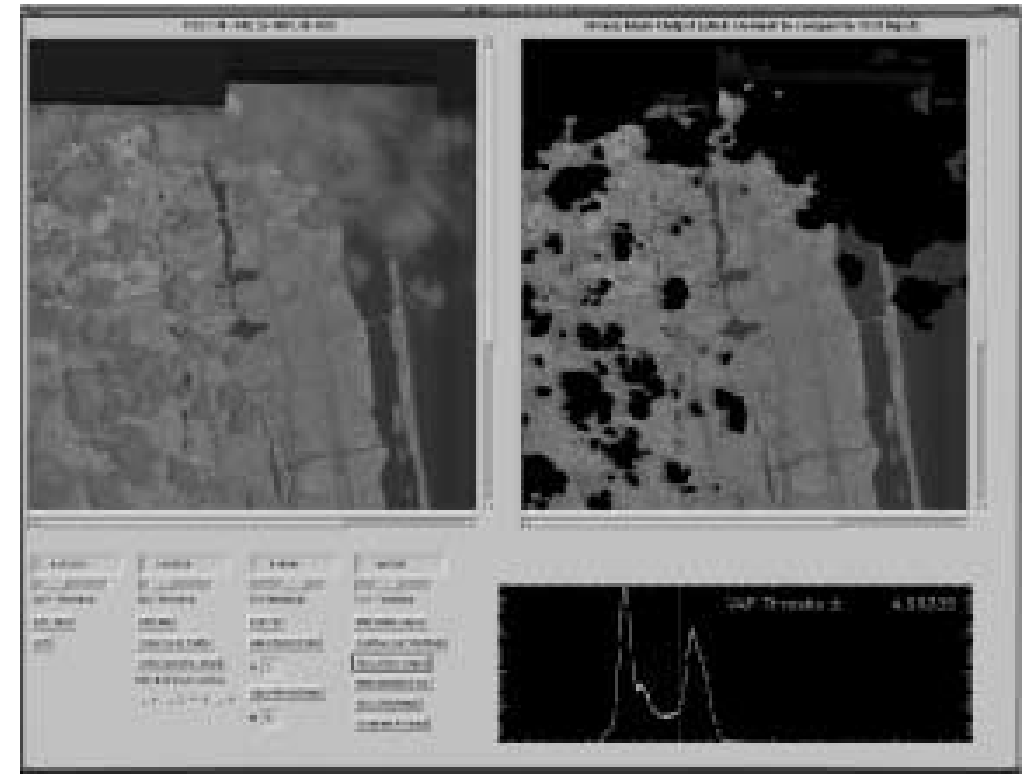

Figure 4. Final step before iteration: overlaying binary cloud mask upon RGB input image.

Figure 3 demonstrates one of four individual mask creations. To assist the analyst there are 3 diagnostic tools. There is an image histogram, a binary mask overlayed upon the single band image, and a superposition of four binary masks encoded to three color channels and intensity. These three images/plots are updated whenever the user changes the current threshold value via a scroll bar, directly typing in, or clicking on the histogram plot.

In figure 5 an example of the 3 color + intensity coded RGB+I cloud mask is shown. In the output window the combination of the individually created threshold masks is shown, with each mask written to a different color channel. The NDVI mask is red, RED mask is green, the TIR mask is blue, and the CIBR water vapor mask is again used normalize intensity. This allows the analyst to immediately see how all of the single masks combine.

Figure 6 is the color truth table. This table shows all possible mask combinations to the analyst using this table the analyst can determine what parts of the RGB combination mask will be marked as true in the final binary cloud mask. In addition the analyst can use this table in conjunction with the $\chi$ parameter to allow or disallow specific individual mask combinations.

Once the analyst is satisfied with the spectral cloud masks, they can operate upon the binary mask with an NxN erode function and an MxM dilate function. This separation and sizing of morphological filters allows for the removal of mask artifacts of differing sizes per image. One final feature of the cloud mask interface (figure 4) is overlaying the binary cloud mask upon the original high cloud contrast RGB image. This is a final check of the cloud masks effectiveness. Since the entire GUI is event-driven this allows the user to proceed in any order, and restart at any phase of the process. Once the analyst is content with the results of the cloud mask overlay, a binary cloud mask product is generated. We estimate that after a short training period the whole process should take no more than five minutes

\section{ANALYST INTERCOMPARISON}

\subsection{Cloud mask comparison}

In order to determine the reliability of this interactive approach we have had several analysts independently create cloud masks for several of the same images. Most of the analyst had no prior training creating cloud masks with other images. As shown in figure 7 there was still little variation in total percent cloud cover between analysts for most images. Upon discussing the large amounts of variation in the Biscayne Bay image with the analysts, it was found that semi-transparent cirrus was sometimes counted as dense cloud and therefore erroneously flagged. 


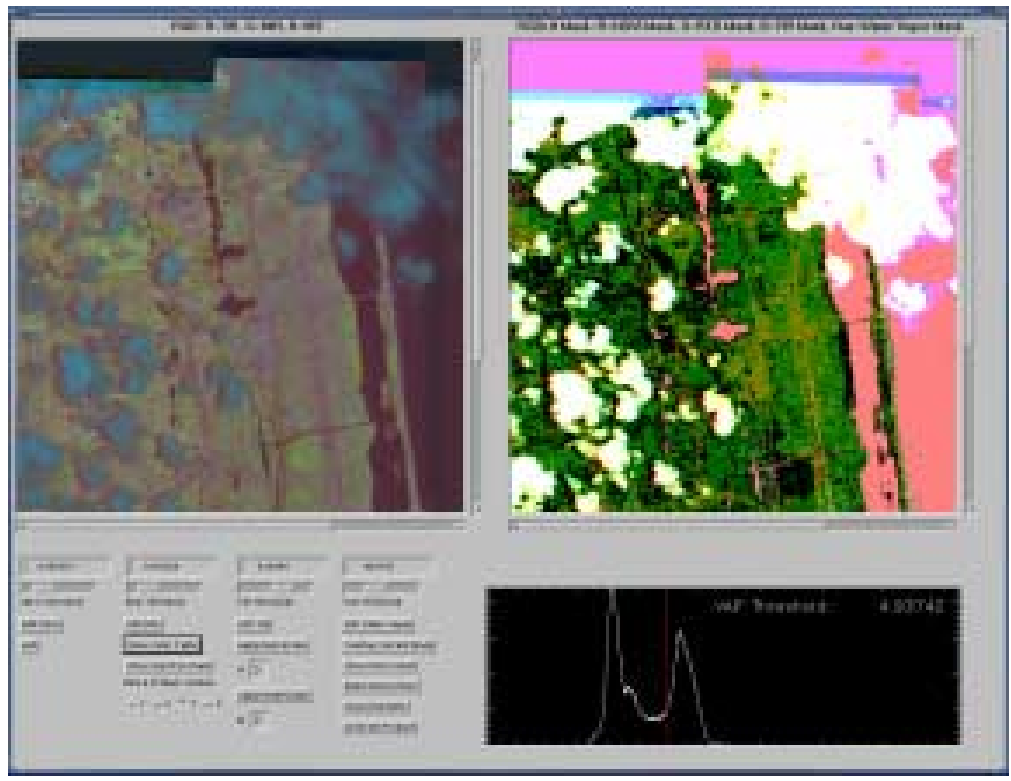

Figure 5. RGB $+\mathrm{I}$ output mask. $\mathrm{R}=$ NDVI mask, $\mathrm{G}=\mathrm{RED}$ mask, $\mathrm{B}=$ TIR mask, Intensity $=$ CIBR Water Vapor mask.

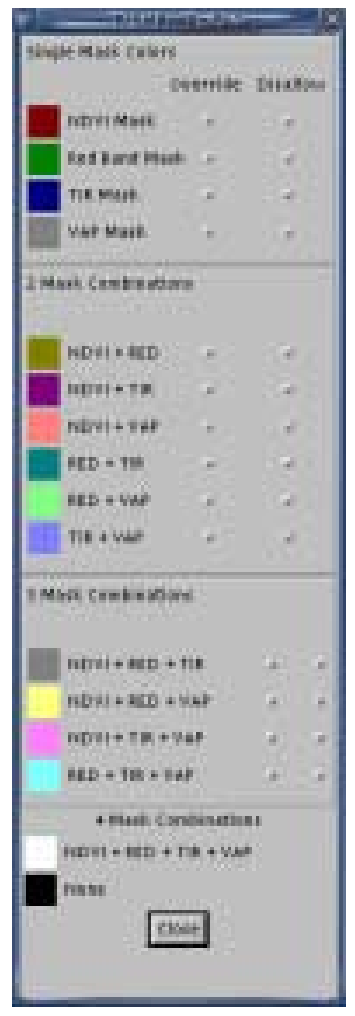

Figure 6. Truth table used to show all possible mask combinations and to override $\chi$ parameter, for specific mask combinations. 


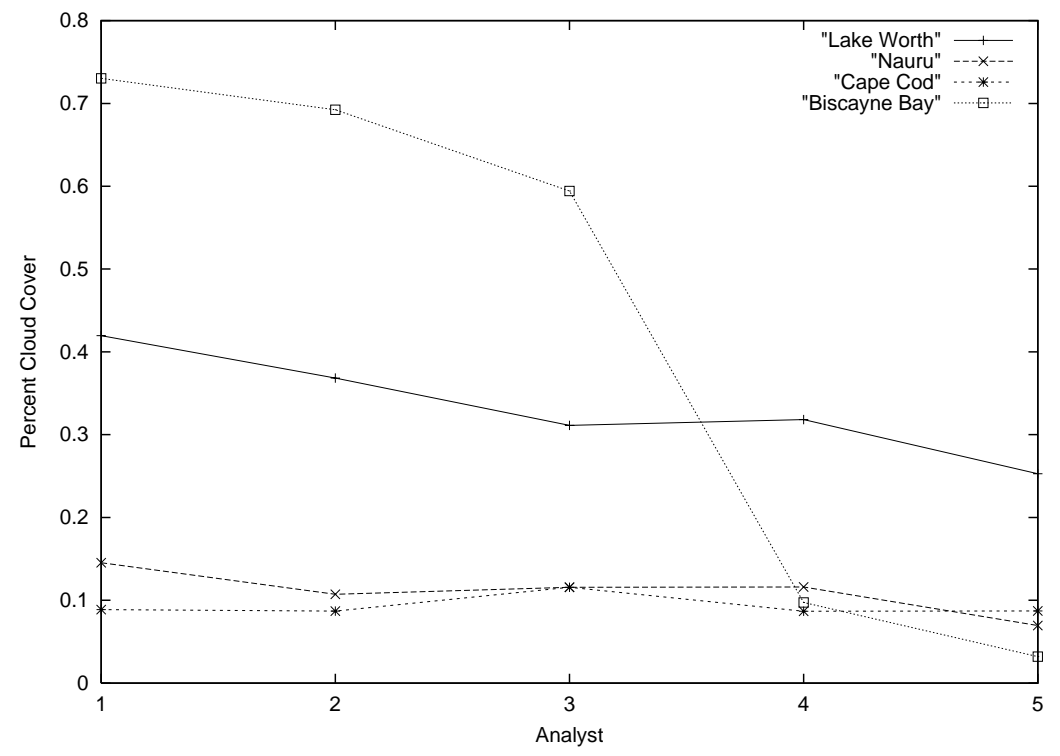

Figure 7. Percent cloud cover found in cloud mask for four different images and five different analysts.

\begin{tabular}{|c|c|c|c|c|c|}
\hline Analyst & 1 & 2 & 3 & 4 & 5 \\
\hline NDVI & 0.3 & 0.235 & 0.201666 & 0.351666 & 0.17 \\
\hline RED & 0.159422 & 0.206394 & 0.0896741 & 0.176503 & 0.239133 \\
\hline TIR & 9.2163 & 9.19267 & 9.19267 & 9.14541 & 9.05088 \\
\hline Water Vap & 5.19669 & 4.93741 & 4.65219 & 4.88556 & 4.93741 \\
\hline Cloud Cover & 0.419611 & 0.368454 & 0.311299 & 0.318156 & 0.252799 \\
\hline \multicolumn{6}{|c|}{ Image 2: Nauru } \\
\hline $\begin{array}{l}\text { NDVI } \\
\end{array}$ & 0.17 & 0.231666 & 0.21 & 0.273333 & 0.138333 \\
\hline RED & 0.500114 & 0.0941386 & 0.0804102 & 0.0764881 & 0.187297 \\
\hline TIR & 9.5476 & 9.46494 & 9.48559 & 9.46494 & 9.58893 \\
\hline Water Vap & 1.38111 & 1.49449 & 4.82531 & 5.12962 & 1.60787 \\
\hline Cloud Cover & 0.145459 & 0.107264 & 0.115801 & 0.116087 & 0.0696303 \\
\hline \multicolumn{6}{|c|}{ Image 3: Cape Cod } \\
\hline NDVI & 0.201666 & 0.056666 & 0.21 & 0.526666 & 0.108333 \\
\hline RED & 0.103418 & 0.344188 & 0.0804102 & 0.347421 & 0.287632 \\
\hline TIR & 7.8612 & 7.47031 & 9.48559 & 7.47031 & 7.68747 \\
\hline Water Vap & 3.21683 & 2.36561 & 4.82531 & 2.47244 & 2.99946 \\
\hline Cloud Cover & 0.0888115 & 0.0868994 & 0.115801 & 0.0867878 & 0.0870837 \\
\hline \multicolumn{6}{|c|}{ Image 4: Biscayne Bay } \\
\hline NDVI & 0.466666 & 0.064516 & 0.316666 & 0.428333 & 0.186666 \\
\hline RED & 0.131233 & 0.240863 & 0.11992 & 0.250023 & 0.221739 \\
\hline TIR & 18.4721 & 9.29506 & 9.22469 & 5.883 & 9.12465 \\
\hline Water Vap & 5.3821 & -1 & 4.97981 & 5.74786 & 4.74207 \\
\hline Cloud Cover & 0.730207 & 0.692443 & 0.594221 & 0.0973168 & 0.0319586 \\
\hline
\end{tabular}

Table 1. This table shows the threshold results of five analysts creating cloud masks for four different images. The -1 in the final test image is an indicator that the analyst set all pixels in the DM to one (true). 


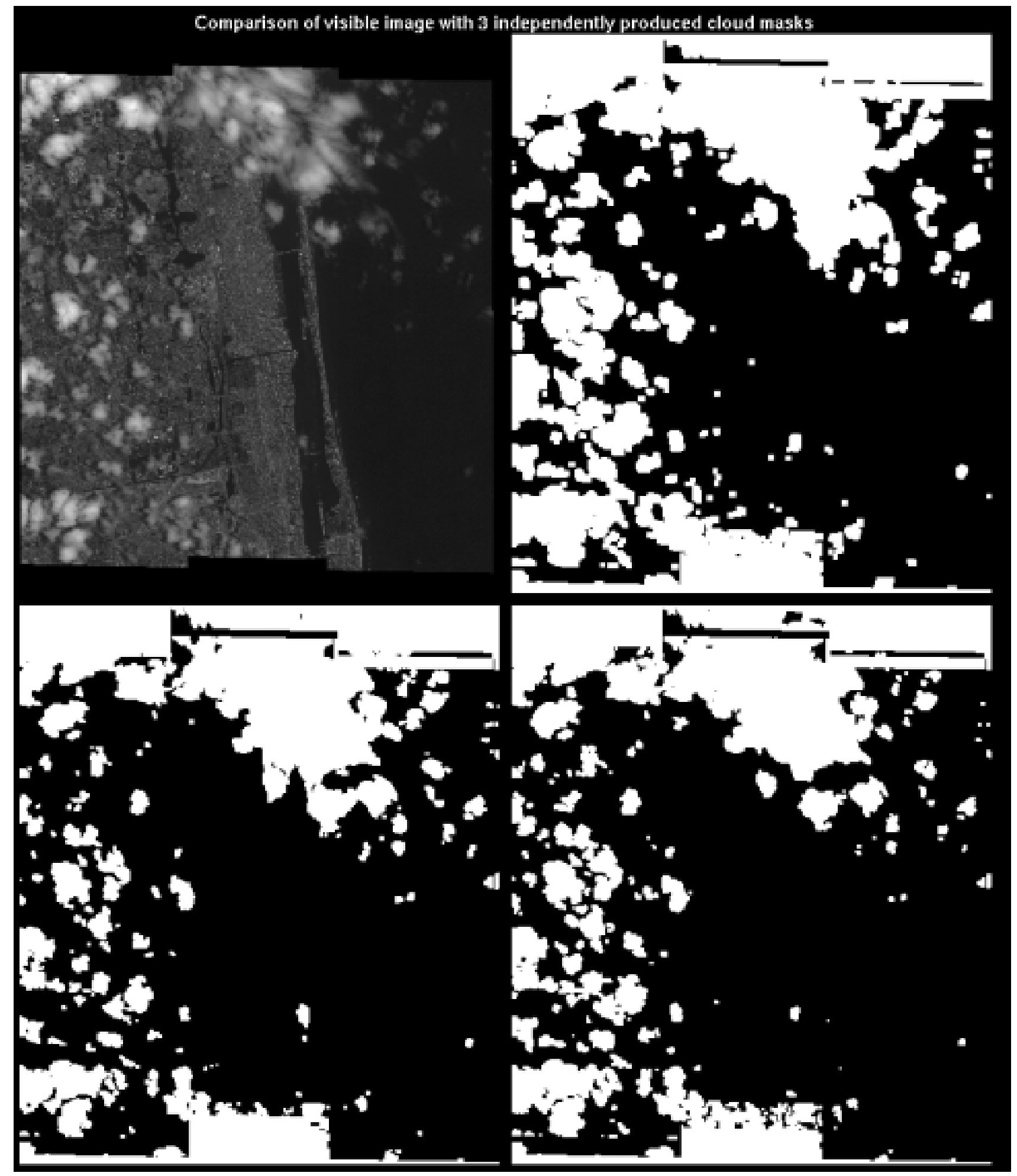

Figure 8. Comparison of 3 different cloud masks for the Lake Worth test image. 
In figure 8 we show a direct comparison of three cloud masks for a single image. This is a comparison of three different analyst's results. There is a progression in cloud masks from very conservative (ie. all cloud edges plus the greatest amount of false positives) to a least conservative (ie. most of the very dense cloud masked and the least number of false positives). Table 1 and figure 7 show this order tends to remain consistent throughout the different images used.

\section{SUMMARY}

We have presented a simple and effective scheme for cloud mask detection using an interactive GUI. Both spectral thresholding and spatial filtering are performed in a manner that promotes consistent cloud mask selection between human analysts, while limiting the amount of effort needed to produce an accurate cloud mask. We have shown that although there are differences between different human analysts, these tend to be small and systematic. Therefore, they can be further reduced through training. This training should demonstrate how conservative to be in the cloud mask creation and provide understandable guidelines on the presence of cirrus in the image.

\section{ACKNOWLEDGMENTS}

This work was supported by the U.S. Department of Energy under Contract W-740-ENG-36 and by the Department of Defense. The authors would like to thank Steven Brumby and Simon Perkins for their assistance with morphological filters. We also thank Emily Riddle and Kim Pollock for their assistance with the creation of cloud masks.

\section{REFERENCES}

1. P. G. Weber, B. C. Brock, A. J. Garrett, B. W. Smith, C. C. Borel, W. B. Clodius, S. C. Bender, R. R. Kay, and M. L. Decker, "Multispectral Thermal Imager mission overview," Proceedings of SPIE - The International Society for Optical Engineering 3753, pp. 394-402, 1999.

2. W. B. Clodius, P. G. Weber, C. C. Borel, and B. W. Smith, "Multi-spectral band selection for satellite-based systems," Proc. SPIE 3377, pp. 11-21, 1998.

3. K. Hirsch, S. P. Brumby, N. R. Harvey, and A. B. Davis, "The MTI dense-cloud mask algorithm to a cloud mask evolved by a genetic algorithm and to the modis cloud mask." presented at International Symposium on Optical Science and Technology, 30 July - 4 August 2000, San Diego, CA USA.

4. S. P. Brumby, J. Theiler, S. J. Perkins, N. R. Harvey, J. J. Szymanski, J. J. Bloch, and M. Mitchell, "Investigation of feature extraction by a genetic algorithm," Proc. SPIE 3812, pp. 24-31, 1999.

5. S. A. Ackerman, K. I. Strabala, W. P. Menzel, R. A. Frey, C. C. Moeller, and L. E. Gumley, "Discriminating clear sky from clouds with MODIS," Journal of Geophysical Research 103, pp. 32141-32158, 1998.

6. C. C. Borel and W. B. Clodius, "Recipes for writing algorithms for atmospheric corrections and temperature/emissivity separations in the thermal regime for a multispectral sensor." presented at SPIE 15th Annual International Symposium on Aerospace/Defence Sensing, Simulation and Controls, Orlando, FL, USA.

7. S. Perkins Private Communication, 26 Feb 2001. 